\title{
Reachability Problems in Nondeterministic Polynomial Maps on the Integers ${ }^{\star}$
}

\author{
Sang-Ki Ko ${ }^{1}$, Reino Niskanen ${ }^{2}$, and Igor Potapov ${ }^{3}$ \\ 1 Korea Electronics Technology Institute, South Korea \\ 2 Department of Computer Science, University of Oxford, UK \\ 3 Department of Computer Science, University of Liverpool, UK
}

\begin{abstract}
We study the reachability problems in various nondeterministic polynomial maps in $\mathbb{Z}^{n}$. We prove that the reachability problem for very simple three-dimensional affine maps (with independent variables) is undecidable and is PSPACE-hard for two-dimensional quadratic maps. Then we show that the complexity of the reachability problem for maps without functions of the form $\pm x+b$ is lower. In this case the reachability problem is PSPACE-complete in general, and NP-hard for any fixed dimension. Finally we extend the model by considering maps as language acceptors and prove that the universality problem is undecidable for two-dimensional affine maps.
\end{abstract}

\section{Introduction}

Many iterative maps can exhibit complex and unpredictable dynamics. They appear in various parts of mathematics and in particular they have been extensively studied in the context of chaos theory $[5,7]$ and control theory $[3,4]$ where these maps have been defined over rational, real or complex numbers. On the other hand iterative maps over integers are also important in computer science as they can be seen as the simplest form of computer programs describing updates on integer counters or variables. The classical reachability problems (i.e., whether a given set of values in the counters/variables can be reached via iterations in loops) are in the core of verification procedures and the complexity of their solutions could vary depending on several factors such as the type of iterative functions (affine, linear, polynomial, elementary, etc.), the form of maps (i.e., deterministic, nondeterministic), the number of variables (i.e., dimension of a system) and even history dependence (i.e., when the next value depends on several previous values of counters/variables) $[14,15]$.

In this paper we study the decidability of the reachability problem for simple stateless systems of nondeterministic iterative polynomial maps defined on integer valued vectors. The simplest form of a map with polynomial updates, i.e., where the updates are of the form $\boldsymbol{x}:=\boldsymbol{x}+\boldsymbol{b}$, can be seen as a vector addition systems (VAS) on $\mathbb{Z}^{n}$. If $n=1$, the reachability problem in one-dimensional system (i.e.,

\footnotetext{
* This work was supported by EPSRC grant "Reachability problems for words, matrices and maps" (EP/M00077X/1).
} 
with one variable/counter) for additive updates of the form $x+b$ can be reduced to the solution of a single linear Diophantine equation over natural numbers and generalization to multidimensional case which is in the form of the $n$-dimensional vector addition system on $\mathbb{Z}^{n}$ is known to be NP-complete [8].

A simple generalization to two-dimensional system of affine updates instead of additive maps makes the reachability problem undecidable over rational numbers $\mathbb{Q}^{2}$; see [1]. The affine transformations that can encode the Post correspondence problem (PCP) are of a very restricted form $p(x, y)=\left(q_{1} x+q_{2} y+q_{3}, q_{4} y\right)$.

Taking it into account we are focusing on a natural generalization by defining multidimensional system with $n$-variables over $\mathbb{Z}^{n}$, where each coordinate dependent only on one variable and then we study the complexity of the reachability problem for different types of the polynomial updates of the form

$$
p\left(x_{1}, \ldots, x_{n}\right)=\left(p_{1}\left(x_{1}\right), \ldots, p_{n}\left(x_{n}\right)\right)
$$

for some univariate polynomials $p_{i}(x)$.

Our research is revealing many surprising complexity results. For example even a simple increase from additive to affine updates where each variable is only self-dependent leads to undecidability of the reachability problem in $\mathbb{Z}^{3}$. The core element of the proof is in the construction of affine functions that simulate a state structure and can be used to control the order of affine updates. Then generalizing the construction to encode any graph structure in one dimension we show that in two-dimensional system with quadratic polynomial functions the reachability is at least PSPACE-hard while we do not know whether in this case the problem is decidable or not; see Table 1.

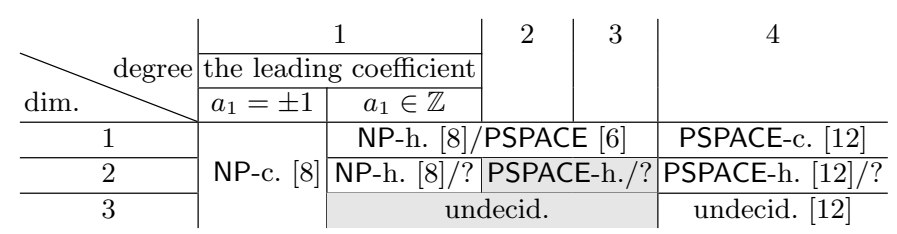

Table 1. Complexity of reachability problems in nondeterministic polynomial maps according to the degrees. Our results are on grey background.

Then we investigate the restrictions on the functions further by considering maps without functions of the form $\pm x+b$. Surprisingly, already for affine maps, this leads to PSPACE upper bound and NP-hardness for any fixed dimension as well as PSPACE-hardness, and thus PSPACE-completeness, if the dimension is not fixed. This heavily contrasts our knowledge on general maps, where most notably, dimension two has no known upper bounds; see Table 2.

Finally, we take a more language-theoretic approach and consider maps as language acceptors. To this end, we fix the initial and target values, $\boldsymbol{z}_{0}$ and $\boldsymbol{z}_{f}$, in the reachability problem for polynomial maps. Then, we attach a letter over a finite alphabet $\Sigma$ to each function. Then, a word $w \in \Sigma$ is accepted if there is a computation path from $\boldsymbol{z}_{0}$ to $\boldsymbol{z}_{f}$ reading $w$ in the map. From this point 


\begin{tabular}{|c|c|c|c|c|}
\hline \multirow{2}{*}{ dim. type } & \multicolumn{2}{|r|}{ affine } & \multicolumn{2}{|c|}{ polynomial } \\
\hline & $a_{1} \neq \pm 1$ & $a_{1} \in \mathbb{Z}$ & not including $\pm x+b$ & including $\pm x+b$ \\
\hline 1 & \multirow{4}{*}{ NP-h./PSPACE } & NP-h. [8]/PSPACE [6] & \multirow{4}{*}{ NP-h./PSPACE } & PSPACE-c. [12] \\
\hline 2 & & NP-h. $[8] / ?$ & & PSPACE-h. [12]/? \\
\hline 3 & & \multirow{2}{*}{ undecid. } & & \\
\hline & & & & \\
\hline
\end{tabular}

Table 2. Complexity of reachability problems in affine and polynomial maps with respect to inclusion of polynomials of the form $\pm x+b$. Our results are on grey background.

of view, the reachability problem is the language emptiness problem. We study another natural language-theoretic question known as the universality problem, where we are asked whether all finite words are accepted by the map. We show that for two-dimensional affine maps the universality problem is undecidable by simulating an integer weighted automaton [9].

The research work on such systems with polynomial updates attracted visible attention in verification community indicating the lack of understanding of even such simple systems. In contrast to stateless systems (iterative maps) a model of polynomial register machines which have additional state structure has been studied in $[6,13]$. The authors showed that the reachability problem is PSPACEcomplete for one-dimensional polynomials and undecidable for two-dimensional polynomials, respectively. Following [6], it was shown in [12] that the reachability problem is PSPACE-complete in polynomial maps of degree four, i.e., stateless machines and it is undecidable for three-dimensional polynomial maps. In [12], the state structure and state transitions were encoded as solutions to systems of linear congruences, where the uniqueness of the solution was guaranteed by the Chinese remainder theorem (similar to [6]). In our paper we give a new proof for the undecidability of the reachability problem for three-dimensional maps where the updates are of a much simpler form of affine polynomials.

Also our approach to look at the reachability from language-theoretic point of view is in line with a very recent paper [2] on the complexity of the zeroness problem for deterministic polynomial automata over rationals (i.e., a problem whether a given automaton outputs zero on all words). Authors of [2] showed that while this problem is non-primitive recursive in general, there is a subclass of polynomial automata for which the zeroness problem is primitive recursive.

\section{Preliminaries}

We denote the set of natural numbers, integers and rational numbers by $\mathbb{N}, \mathbb{Z}$ and $\mathbb{Q}$, respectively. The integers are assumed to be encoded in binary. For $z_{1} \leq z_{2} \in \mathbb{Z}$, we denote the closed interval by $\left[z_{1}, z_{2}\right]=\left\{z \in \mathbb{Z} \mid z_{1} \leq z \leq z_{2}\right\}$.

We denote the ring of polynomials with integer coefficients over a variable $x$ by $\mathbb{Z}[x]$. The multidimensional polynomials $\mathbb{Z}[\boldsymbol{x}]^{n}$ with independent variables $\boldsymbol{x}=\left(x_{1}, \ldots, x_{n}\right)$ are of the form $p\left(x_{1}, \ldots, x_{n}\right)=\left(p_{1}\left(x_{1}\right), \ldots, p_{n}\left(x_{n}\right)\right)$ for some univariate polynomials $p_{1}(x), \ldots, p_{n}(x) \in \mathbb{Z}[x]$. That is, dimensions are indepen- 
dent and do not affect the values in other dimensions. Throughout the paper, we investigate the reachability problem for different classes of polynomials and in order to simplify the terminology, we give names to the commonly used classes:

Additive polynomials:

Affine polynomials:

Quadratic polynomials:

$$
\begin{aligned}
\operatorname{Add}_{\mathbb{Z}} & =\{ \pm x+b \mid b \in \mathbb{Z}\} \\
\operatorname{Aff}_{\mathbb{Z}}[x] & =\{a x+b \mid a, b \in \mathbb{Z}\}, \\
\operatorname{Quad}_{\mathbb{Z}}[x] & =\left\{a x^{2}+b x+c \mid a, b \in \mathbb{Z}\right\} .
\end{aligned}
$$

We also define the classes of polynomials without additive polynomials ${ }^{4}$, i.e., updates of form $\pm x+b$ :

$$
\begin{aligned}
\operatorname{Aff}_{\mathbb{Z}}[x] \backslash \operatorname{Add}_{\mathbb{Z}} & =\left\{a x+b \in \operatorname{Aff}_{\mathbb{Z}}[x] \mid a \neq \pm 1\right\}, \\
\mathbb{Z}[x] \backslash \operatorname{Add}_{\mathbb{Z}} & =\{p(x) \in \mathbb{Z}[x] \mid p(x) \neq \pm x+b, \text { where } b \in \mathbb{Z}\} .
\end{aligned}
$$

The multidimensional variants $\operatorname{Aff}_{\mathbb{Z}}[\boldsymbol{x}]^{n}$ and $\operatorname{Quad}_{\mathbb{Z}}[\boldsymbol{x}]^{n}$ are defined in the natural way, while $\operatorname{Aff}_{\mathbb{Z}}[\boldsymbol{x}]^{n} \backslash \operatorname{Add}_{\mathbb{Z}}$ is defined as $\left(\operatorname{Aff}_{\mathbb{Z}}[x] \backslash \operatorname{Add}_{\mathbb{Z}}\right) \times \ldots \times\left(\operatorname{Aff}_{\mathbb{Z}}[x] \backslash \operatorname{Add}_{\mathbb{Z}}\right)$ and $\mathbb{Z}[\boldsymbol{x}]^{n} \backslash \operatorname{Add}_{\mathbb{Z}}$ is defined analogously. The class $\mathbb{Z}[x] \backslash \operatorname{Add}_{\mathbb{Z}}$ seems artificial at first but, as we prove later, polynomials of the form $\pm x+b$ play a vital role in whether the reachability problem is decidable or undecidable. Indeed, we will show that for $\mathbb{Z}[\boldsymbol{x}]^{n} \backslash \operatorname{Add}_{\mathbb{Z}}$, the reachability problem is in PSPACE, while the problem is undecidable already for $\operatorname{Aff}_{\mathbb{Z}}[\boldsymbol{x}]^{3}$. Note that the identity function $p(x)=x$ is also classified as a function in $\mathbb{Z}[x] \backslash \operatorname{Add}_{\mathbb{Z}}$. The different classes of polynomials are depicted below:

$$
a_{n} x^{n}+\ldots+\overbrace{a_{2} x^{2}+\underbrace{a_{1} x+a_{0}}_{\in \operatorname{Aff}_{\mathbb{Z}}[x]}}^{\in \operatorname{Quad}_{\mathbb{Z}}[x]} \in \mathbb{Z}[x] \quad \pm x+a_{0} \in \operatorname{Add}_{\mathbb{Z}}
$$

An $n$-dimensional polynomial register machine $(n$-PRM) is a tuple $\mathcal{R}=(Q, \Delta)$, where $Q$ is a finite set of states and $\Delta \subseteq Q \times \mathbb{Z}[\boldsymbol{x}]^{n} \times Q$ is a finite set of transitions labelled by polynomials with variable $\boldsymbol{x} \in \mathbb{Z}^{n}$. A configuration of $\mathcal{R}$ is a tuple $[q, \boldsymbol{x}] \in Q \times \mathbb{Z}^{n}$. A configuration $\left[q^{\prime}, \boldsymbol{y}^{\prime}\right]$ is reachable from a configuration $[q, \boldsymbol{y}]$ by a transition $\left(q, f(\boldsymbol{x}), q^{\prime}\right)$ if $f(\boldsymbol{y})=\boldsymbol{y}^{\prime}$. This is denoted by $[q, \boldsymbol{y}] \rightarrow_{\mathcal{R}}\left[q^{\prime}, \boldsymbol{y}^{\prime}\right]$. The reflexive and transitive closure of $\rightarrow_{\mathcal{R}}$ is denoted by $\rightarrow_{\mathcal{R}}^{*}$ The reachability problem is, given two configurations $c$ and $c^{\prime}$ of $\mathcal{R}$, to decide whether $c \rightarrow_{\mathcal{R}}^{*} c^{\prime}$ holds. If an $n$-PRM $\mathcal{R}$ has only one state, then $\mathcal{R}$ is called a nondeterministic polynomial map or a map over $\mathbb{Z}[\boldsymbol{x}]^{n}$ for short.

An $n$-PRM is called an $n$-dimensional affine register machine ( $n$-ARM) if every transition is labelled by an affine polynomial. A nondeterministic affine map (a map over $\operatorname{Aff}_{\mathbb{Z}}[\boldsymbol{x}]^{n}$ ) is defined analogously.

A linear-bounded automaton (LBA) is a Turing machine with a tape bounded by a linear function of the length of the input. In other words, an LBA can be viewed as a Turing machine with a finite tape. We denote an LBA $\mathcal{A}$ by a tuple $(Q, \Gamma, \delta)$, where $Q$ is a finite set of states, $\Gamma=\{\triangleright, \triangleleft, 0,1\}$ is a finite

\footnotetext{
${ }^{4}$ In [6], additive polynomials were called counter-like as they are similar to updates in counter machines and VASSs.
} 
tape alphabet, which includes two special symbols $\triangleright$ and $\triangleleft$ serving as left and right endmarkers of the tape. See for example [10] for more details on LBA. The reachability problem of a given LBA $\mathcal{A}$ is to decide whether for given length of the tape $k$, states $q$ and $q^{\prime},\left(q, \triangleright 0^{k} \triangleleft, 0\right) \rightarrow_{A}^{*}\left(q^{\prime}, \triangleright 0^{k} \triangleleft, 0\right)$ holds and is well-known to be PSPACE-complete.

In the next lemma, we give a function $\sigma$ that outputs a number in tertiary representation of the input word over the binary alphabet $\Sigma$. Since each natural number has a unique tertiary representation, $\sigma$ is clearly an injective function. This function is commonly used in the literature in reductions from the PCP. Recall that the PCP is undecidable for $n_{p}=5$ pairs of words [11].

Lemma 1. Let $\Sigma=\{a, b\}$ and $w \in \Sigma^{*}$. Let $\tau: \Sigma \rightarrow \mathbb{N}$ be defined as $\tau(a)=1$ and $\tau(b)=2$. The function $\sigma: \Sigma^{*} \rightarrow \mathbb{N}$ defined by $\sigma\left(w_{1} w_{2} \cdots w_{k}\right)=\sum_{i=1}^{k} \tau\left(w_{i}\right)$. $3^{k-i}$ and $\sigma(\varepsilon)=0$ is an injective function.

\section{Reachability in maps over $\operatorname{Aff}_{\mathbb{Z}}[x]^{3}$ and $\operatorname{Quad}_{\mathbb{Z}}[x]^{2}$}

In this section, we consider the reachability problem in multidimensional maps. We will show undecidability for $\operatorname{Aff}_{\mathbb{Z}}[\boldsymbol{x}]^{3}$ and PSPACE-hardness for Quad ${ }_{\mathbb{Z}}[\boldsymbol{x}]^{2}$ when the coefficients of the functions are integers. That is, in the three-dimensional variant, we are investigating functions of the form

$$
\left\{\begin{array}{l}
x_{1}:=a_{1} x_{1}+b_{1} \\
x_{2}:=a_{2} x_{2}+b_{2} \\
x_{3}:=a_{3} x_{3}+b_{3}
\end{array},\right.
$$

where $a_{i}, b_{i} \in \mathbb{Z}$, and show that the reachability problem is undecidable by encoding the PCP in the first two dimensions and using the third dimension to make sure that only one word is constructed. It is important to note that the reachability problem in maps over $\operatorname{Aff}_{\mathbb{Z}}[\boldsymbol{x}]^{3}$ is undecidable only in the case where affine functions can be of the form $\pm x+b$. As we will prove in the following section, the reachability problem is decidable even for polynomials of any degree, as long as none is of the form $\pm x+b$, i.e., for maps over $\mathbb{Z}[\boldsymbol{x}]^{n} \backslash \operatorname{Add}_{\mathbb{Z}}$ for any $n$. Finally, we will consider two-dimensional maps, where the polynomials are quadratic and show that the reachability problem is PSPACE-hard.

Theorem 2. The reachability problem for maps over $A f_{\mathbb{Z}}[\boldsymbol{x}]^{3}$ is undecidable with at least $n_{p}+2=7$ affine functions over $\mathbb{Z}$.

Proof. Let $P=\left\{\left(u_{1}, v_{1}\right),\left(u_{2}, v_{2}\right), \ldots,\left(u_{n}, v_{n}\right)\right\} \subseteq \Sigma^{*} \times \Sigma^{*}$ be an instance of the $\mathrm{PCP}$. We define a set of three-dimensional affine functions to simulate the PCP. We show that $(0,0,1)$ is reachable from $(0,0,0)$ if and only if the PCP has a solution. For each pair $\left(u_{i}, v_{i}\right) \in P$, where $1 \leq i \leq n$, we define the following sets of affine functions in dimension three:

$$
\begin{aligned}
& F_{1}=\left\{\left(3^{\left|u_{i}\right|} x_{1}+\sigma\left(u_{i}\right), 3^{\left|v_{i}\right|} x_{2}+\sigma\left(v_{i}\right), 2 x_{3}\right) \mid\left(u_{i}, v_{i}\right) \in P \text { for all } 1 \leq i \leq n\right\}, \\
& F_{2}=\left\{\left(3^{\left|u_{i}\right|} x_{1}+\sigma\left(u_{i}\right), 3^{\left|v_{i}\right|} x_{2}+\sigma\left(v_{i}\right), 2 x_{3}+1\right) \mid\left(u_{i}, v_{i}\right) \in P \text { for all } 1 \leq i \leq n\right\}, \\
& F_{3}=\left\{\left(x_{1}-1, x_{2}-1,2 x_{3}-1\right)\right\},
\end{aligned}
$$




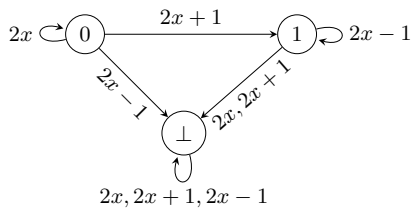

Fig. 1. An illustration of the behaviour of the register. State $\perp$ corresponds to register value being other than 0 or 1 .

where $\sigma$ is the embedding of Lemma 1.

Consider the two configurations $(0,0,0)$ and $(0,0,1)$. We prove the undecidability of the reachability problem in affine map by showing that the configuration $(0,0,1)$ is reachable from the initial configuration $(0,0,0)$ by applying affine functions in $F=F_{1} \cup F_{2} \cup F_{3}$ if and only if the PCP instance $P$ has a solution.

First, we prove that a configuration $(x, y, 1)$, for some $x, y \in \mathbb{Z}$, is reachable from $(0,0,0)$ if and only if first functions from $F_{1}$ are applied, then a function from $F_{2}$, and finally functions from $F_{3}$ are applied. Observe first that affine functions in the third components in the functions from $F_{1}, F_{2}$ and $F_{3}$ have fixed points $0,-1$ and 1 , respectively. Additionally, if $x$ is not a fixed point of a function $p(x)$ from some $F_{i}$, then $|p(x)|>|x|$. Now it is easy to see that applying the functions in the claimed order results in third component being 1 . Indeed, first 0 is fixed by applying functions from $F_{1}$, then it is mapped to $2 \cdot 0+1=1$ by a function from $F_{2}$, and finally is again fixed by functions from $F_{3}$. If the functions are applied in a different order, then, as the absolute value does not decrease, value 1 cannot be reached. This is illustrated in Figure 1.

Now we are ready to prove that we can reach both zeros in the first and second dimensions if and only if the PCP has a solution. It is possible to construct an identical pair of words by concatenating the pairs in the PCP instance $P$ if and only if the PCP has a solution. In other words, we can reach a configuration $(y, y, 1)$ for some $y \in \mathbb{N}$ by applying the functions in $F_{1}$ and $F_{2}$ if and only if we have a solution for the PCP instance $P$. Then, it is easy to see that the target configuration $(0,0,1)$ is reachable by applying the only affine function in $F_{3}$ if and only if the PCP has a solution. Recall that the PCP is undecidable for $n=5$ pairs [11]. Note that $|F|=2 n+1=11$. To achieve the claimed size of 7 , we observe that since a function from $F_{2}$ is applied only once, we can consider a set

$$
F_{2}^{i}=\left\{\left(3^{\left|u_{i}\right|} \cdot x_{1}+\sigma\left(u_{i}\right), 3^{\left|v_{i}\right|} \cdot x_{2}+\sigma\left(v_{i}\right), 2 \cdot x_{3}+1\right)\right\},
$$

for each $1 \leq i \leq n$. Now the reachability problem is undecidable for some $F_{1} \cup F_{2}^{i} \cup F_{3}$ and there are 7 functions in the set.

In the previous proof, a state structure was simulated by affine functions. Next we generalize the construction to simulate an arbitrary state structure. We use this result in upcoming results of the paper to embed a state structure of an automaton into affine functions. It will allow us to prove PSPACE-hardness for 
$\operatorname{maps}$ over $\operatorname{Quad}_{\mathbb{Z}}[\boldsymbol{x}]^{2}$ and undecidability of the universality problem for maps over $\operatorname{Aff}_{\mathbb{Z}}[\boldsymbol{x}]^{2}$.

Lemma 3. Let $G=(V, E)$ be a directed graph with vertices $v_{0}, \ldots, v_{m-1}$. There exists a set of affine functions $F$ such that for an edge $\left(v_{i}, v_{j}\right) \in E$, where $0 \leq i, j \leq m-1$, there exists a unique $f_{i j} \in F$ such that $f_{i j}(i)=j$ and $f_{i j}(x) \notin[0, m-1]$ for all $x \neq i$.

Proof (Sketch). Let $G=(V, E)$ be a graph with $V=\left\{v_{0}, \ldots, v_{m-1}\right\}$. For each edge $\left(v_{i}, v_{j}\right)$ (possibly $i=j$ ) of $G$, we add an affine polynomial $f_{i j}(x)=m(x-i)+j$ to the map. The idea is for integers in $[0, m-1]$ to represent each vertex and with the correct affine function, the value of the register changes according to the graph. In the event of wrong affine function being chosen, the coefficient $m$ ensures that the resulting value of the register will be either larger than $m$ or less than zero. Further, in both cases, none of the subsequent functions result in a value in $[0, m-1]$.

Observe that Lemma 3 gives us a correspondence between an edge in a graph and an affine function. It is easy to see that there is a one-to-one correspondence between a path from vertex $v_{i}$ to vertex $v_{j}$ in the graph and a sequence of affine functions transforming $i$ into $j$. We can use Lemma 3 to embed a state structure into a one-dimensional affine map. In the next theorem, we embed a 1-PRM of [6] into a two-dimensional map, where the functions are at most quadratic.

Theorem 4. The reachability problem for maps over $\operatorname{Quad}_{\mathbb{Z}}[\boldsymbol{x}]^{2}$ is PSPACE-hard.

Proof. Let $\mathcal{R}=(Q, \Delta)$ be a one-dimensional PRM with PSPACE-hard reachability problem [6]. Denote $Q=\left\{q_{0}, \ldots, q_{m-1}\right\}$. Note that the update polynomials of $\mathcal{R}$ are quadratic [6]. For each transition $\left(q_{i}, p(x), q_{j}\right)$ of $\mathcal{R}$, we add two-dimensional function $(p(x), m \cdot x+j-m \cdot i)$ to the map. It is clear that $(0, k)$ is reachable from $(0, \ell)$ if and only if $\left[q_{\ell}, 0\right] \rightarrow_{\mathcal{R}}^{*}\left[q_{k}, 0\right]$. That is, the reachability problem for $\operatorname{maps}$ over $\operatorname{Quad}_{\mathbb{Z}}[\boldsymbol{x}]^{2}$ is PSPACE-hard.

There are two main open problems for dimension two. Namely, whether the reachability problem for maps over $\operatorname{Aff}_{\mathbb{Z}}[\boldsymbol{x}]^{2}$ or $\mathbb{Z}[\boldsymbol{x}]^{2}$ are decidable.

\section{Reachability in maps without additive updates}

In this section, we consider a restricted class of maps over $\operatorname{Aff}_{\mathbb{Z}}[x]$, in the sense that every affine function in the map is not of the form $\pm x+b$. It is easy to see that the reachability problem for maps over $\operatorname{Aff}_{\mathbb{Z}}[\boldsymbol{x}]^{n}$ is NP-hard starting from dimension one as we can easily reduce the subset sum problem (SSP) to the reachability problem in maps. The NP-hardness proof relies on the use of polynomials of the form $x+b$ that correspond to integers in the SSP. Then, we can easily reduce the SSP with a target integer $s$ to the reachability problem for maps over $\mathrm{Aff}_{\mathbb{Z}}[x]$ with a target register $s$. If we further restrict ourselves to maps where all polynomials are of the form $\pm x+b$, then the reachability problem 
is NP-complete [8]. However, we do not have a tight complexity bound for the reachability problem for maps over $\operatorname{Aff}_{\mathbb{Z}}[x]$ to the best of our knowledge. The best upper bound we know of is the PSPACE upper bound given by Finkel et al. [6] following from the PSPACE-completeness of the reachability problem for one-dimensional PRMs.

We consider a dual setting, where all polynomials of the form $\pm x+b$ are excluded, i.e., maps over $\mathbb{Z}[\boldsymbol{x}]^{n} \backslash \operatorname{Add}_{\mathbb{Z}}$. First we prove that the reachability problem for maps over $\mathbb{Z}[\boldsymbol{x}]^{n} \backslash \operatorname{Add}_{\mathbb{Z}}$ is in PSPACE for any dimension $n$. Then, we establish the PSPACE-completeness for maps over $\operatorname{Aff}_{\mathbb{Z}}[\boldsymbol{x}]^{n} \backslash \operatorname{Add}_{\mathbb{Z}}$, when $n$ is not fixed, by proving the hardness via a reduction of the reachability problem for LBA. Let us first prove that the reachability problem for maps over $\operatorname{Aff}_{\mathbb{Z}}[x] \backslash \operatorname{Add}_{\mathbb{Z}}$ remains NP-hard. That is, we give an alternative proof for NP-hardness of the reachability problem for maps over $\operatorname{Aff}_{\mathbb{Z}}[x]$ that does not rely on additive updates.

Lemma 5. The reachability problem for maps over $A f f_{\mathbb{Z}}[x] \backslash A d d_{\mathbb{Z}}$ is NP-hard.

Proof (Sketch). Let $(S, s)$ be an instance of the SSP, where $S=\left\{s_{1}, \ldots, s_{k},\right\}$ and $s$ is the target integer. We construct the set of affine functions $F=\{n \cdot x+$ $\left.n^{i-1} \cdot s_{i}, n \cdot x \mid 1 \leq i \leq k\right\}$ with target $n^{k-1}$, where $n>\max (S) \cdot|S|$ is a prime. We leave as an exercise to verify that the map reaches $n^{k-1}$ if and only if there is a subset of $S$ such that its elements add up to $s$.

Like the case of maps over $\mathrm{Aff}_{\mathbb{Z}}[x]$, we know that the reachability problem for maps over $\mathrm{Aff}_{\mathbb{Z}}[x] \backslash \operatorname{Add}_{\mathbb{Z}}$ is decidable in PSPACE and is NP-hard. However, unlike the general case of affine maps where the problem becomes undecidable in higher dimensions, the reachability problem for maps over $\operatorname{Aff}_{\mathbb{Z}}[\boldsymbol{x}]^{n} \backslash \operatorname{Add}_{\mathbb{Z}}$ stays in PSPACE for any dimension. The reachability problem remains in PSPACE even if we do not impose a limit on the degree of polynomials, that is, for $\mathbb{Z}[\boldsymbol{x}]^{n} \backslash \operatorname{Add}_{\mathbb{Z}}$.

Theorem 6. The reachability problem for maps over $\mathbb{Z}[\boldsymbol{x}]^{n} \backslash A d d_{\mathbb{Z}}$ is decidable in PSPACE for any $n \geq 1$.

Proof. Consider first $n=1$. We follow the reasoning of [6]. There exists a bound $b \in \mathbb{N}$ such that every polynomial in $\mathbb{Z}[x] \backslash \operatorname{Add}_{\mathbb{Z}}$ is monotonically increasing or decreasing in $\mathbb{Z} \backslash[-b, b]$, i.e., $|f(x)|>|x|$ for $x \in \mathbb{Z} \backslash[-b, b]$. Note that $b$ is polynomial in size of the input. Let $z$ be the target integer. If $|z| \leq b$, we can decide whether the integer $z$ is reachable in PSPACE by applying the given functions since we can store the current value and the computation path in space polynomial in $b$ which is again of size polynomial in the input. If $z$ is outside the interval $[-b, b]$, due to monotonicity properties of $\mathbb{Z}[x] \backslash \operatorname{Add}_{\mathbb{Z}}$ functions, we do not need to consider the integers outside the interval $[-z, z]$. That is, still only polynomial space in size of the input is required.

The PSPACE upper bound holds even if we consider $n$-dimensional case since we can maintain the information of the current computation for each dimension in space polynomial in the input.

By considering a larger domain, the undecidability for three-dimensional maps remains, even without additive updates. 
Theorem 7. The reachability problem for rational maps over $A f f_{\mathbb{Q}}[\boldsymbol{x}]^{3} \backslash$ Add $_{\mathbb{Q}}$ is undecidable with at least $2 n_{p}+1=11$ affine functions over $\mathbb{Q}$.

Now we prove the PSPACE-hardness of the reachability problem for maps over $\operatorname{Aff}_{\mathbb{Z}}[\boldsymbol{x}]^{n} \backslash \operatorname{Add}_{\mathbb{Z}}$ by reducing the reachability problem of LBAs to it. Note that the dimension $n$ is not fixed.

Lemma 8. The reachability problem for maps over $A f f_{\mathbb{Z}}[\boldsymbol{x}]^{n} \backslash A d d_{\mathbb{Z}}$ is PSPACEhard.

Proof. Let $\mathcal{A}=(Q, \Gamma, \delta)$, where $Q=\left\{q_{0}, \ldots, q_{m-1}\right\}$, be an LBA with PSPACEhard reachability problem. Recall that in the reachability problem for LBAs, we are asked whether $\left(q_{0}, \triangleright 0^{k} \triangleleft, 0\right) \rightarrow_{\mathcal{A}}^{*}\left(q^{\prime}, \triangleright 0^{k} \triangleleft, 0\right)$ holds. We reduce this reachability problem to the reachability problem for maps over $\operatorname{Aff}_{\mathbb{Z}}[\boldsymbol{x}]^{k+1} \backslash \operatorname{Add}_{\mathbb{Z}}$. Let us define the set $F_{\mathcal{A}}$ of affine functions that simulate the computation of $\mathcal{A}$.

The main idea is that we store the tape content of the LBA $\mathcal{A}$ in the first $k$ dimensions of the affine map. Then, we maintain the information of the current state and the head position in the final dimension using the construction of Lemma 3.

Let $\left(q_{j}, \triangleright w \triangleleft, i\right)$ be the current configuration of $\mathcal{A}$, where $0 \leq j \leq m-1$ and $1 \leq i \leq|w|$. Denote $w=w_{1} w_{2} \cdots w_{k} \in\{0,1\}^{k}$. The corresponding register value in the affine map is $\left(w_{1}, w_{2}, \ldots, w_{k}, z\right)$, where $z$ corresponds to the head being in state $q_{j}$ in position $i$ of the tape.

First, let us construct a state structure incorporating information on both state and position of the head of $\mathcal{A}$. That is, we construct a graph $G_{\mathcal{A}}$ with vertices $(q, i) \in Q \times[1, k]$ and with edges $\left((q, i),\left(q^{\prime}, i-1\right)\right)$ if there is a transition $\left(q, a, q^{\prime}, b, L\right) \in \delta$, and $\left((q, i),\left(q^{\prime}, i+1\right)\right)$ if there is a transition $\left(q, a, q^{\prime}, b, R\right) \in \delta$, for any $a, b \in \Gamma$. Note that $G_{\mathcal{A}}$ does not take the tape content into account. By Lemma 3 , the graph $G_{\mathcal{A}}$ can be simulated by affine functions. We omit details on the behaviour of $\mathcal{A}$ on the endmarkers $\triangleright$ and $\triangleleft$. These transitions can be easily hardcoded into the graph.

Then, to simulate rewriting of a tape, we consider a graph $G_{\Gamma}$ with two vertices, 0 and 1 , and edges are $(0,0),(0,1),(1,0)$ and $(1,1)$. Intuitively, vertex 0 corresponds to the symbol being 0 and edge $(0,0)$ corresponds to the head not rewriting the symbol, while $(0,1)$ means that the symbol was rewritten as 1 . By Lemma 3, there exists a set of affine functions simulating this behaviour.

We are now ready to combine the two sets in order to simulate $\mathcal{A}$. Let us consider the transition $\left(q_{j_{1}}, 0, q_{j_{2}}, 1, L\right) \in \delta$ and the current head position is $i$ where $1 \leq i \leq k$. This transition switches the state from $q_{j_{1}}$ to $q_{j_{2}}$ if the tape has 0 in the current head position while writing 1 and moving the head position to the left. This implies that in $G_{\mathcal{A}}$ constructed previously, we need to take the edge $\left(\left(q_{j_{1}}, i\right),\left(q_{j_{2}}, i-1\right)\right)$ to successfully perform the transition. Further, in the $i$ th dimension, we apply edge $(0,1)$ of $G_{\Gamma}$. Note that we apply the identity function in the other dimensions unless explicitly mentioned. The affine function corresponding to $\left(q_{j_{1}}, 0, q_{j_{2}}, 1, L\right)$ is $(x, \ldots, x, 2 x+1, x, \ldots, x, a \cdot x+b)$, where $a \cdot x+b$ corresponds to the edge $\left(\left(q_{j_{1}}, i\right),\left(q_{j_{2}}, i-1\right)\right)$ in $G_{\mathcal{A}}$, and $2 x+1$ is in the $i$ th dimension. The transitions moving the head to right are defined analogously. 
Since we have proved that every transition of $\mathcal{A}$ can be simulated by an affine function, it is clear that if $\left(q, \triangleright 0^{k} \triangleleft, 0\right) \rightarrow_{\mathcal{A}}^{*}\left(q^{\prime}, \triangleright 0^{k} \triangleleft, 0\right)$ holds in $\mathcal{A}$, then we can reach the register in the affine map corresponding to $\left(q^{\prime}, \triangleright 0^{k} \triangleleft, 0\right)$ from the register corresponding to the initial configuration $\left(q, \triangleright 0^{k} \triangleleft, 0\right)$ of $\mathcal{A}$. Therefore, the reachability problem for maps over $\operatorname{Aff}_{\mathbb{Z}}[\boldsymbol{x}]^{n} \backslash \operatorname{Add}_{\mathbb{Z}}$ is PSPACE-hard.

Based on Theorem 6 and Lemma 8, we have the following main result:

Theorem 9. If the dimension $n$ is not fixed, then the reachability problem for maps over $A f f_{\mathbb{Z}}[\boldsymbol{x}]^{n} \backslash A d d_{\mathbb{Z}}$ is PSPACE-complete.

It is not difficult to see that the PSPACE-completeness holds for multidimensional ARMs and PRMs without additive updates as well.

Corollary 10. If the dimension $n$ is not fixed, then the reachability problem for $n$-ARMs and $n$-PRMs, where the update polynomials are not of the form $\pm x+b$, is PSPACE-complete.

Corollary 11. If the dimension $n$ is not fixed, then the reachability problem for maps over $\mathbb{Z}[\boldsymbol{x}]^{n} \backslash$ Add $_{\mathbb{Z}}$ is PSPACE-complete.

We consider a sort of dual of Lemma 3 by investigating PRMs, where the state structure is induced by affine functions. Let $F \subseteq\left(\operatorname{Aff}_{\mathbb{Z}}[x] \backslash \operatorname{Add}_{\mathbb{Z}}\right) \times \mathbb{Z}[x]$ and $\boldsymbol{x}_{0}$ and $\boldsymbol{x}_{f}$ be the initial and target values respectively. Let $m$ be the largest coefficient in the first component. We construct a graph with $m$ vertices where the transitions are defined by the affine functions and consider it as a PRM. Since the reachability problem for PRMs is in PSPACE, the reachability problem for maps over $\left(\operatorname{Aff}_{\mathbb{Z}}[x] \backslash \operatorname{Add}_{\mathbb{Z}}\right) \times \mathbb{Z}[x]$ is in EXPSPACE as there is exponential number of states.

Theorem 12. The reachability problem for maps over $\left(\operatorname{Aff}_{\mathbb{Z}}[x] \backslash \operatorname{Add}_{\mathbb{Z}}\right) \times \mathbb{Z}[x]$ is in EXPSPACE.

\section{Maps over $\operatorname{Aff}_{\mathbb{Z}}[x]^{2}$ as language acceptors}

In this section, we extend our models to operate on words. Then it is natural to consider the languages accepted by the maps. In this context, the reachability problems of the previous sections can be seen as language emptiness problems. Indeed, the language accepted by the map is empty if and only if the final configuration is not reachable from the initial configuration. The complementary problem to the emptiness problem is the universality problem, where we are asked whether every word is accepted by the computational model. We show that for maps over $\operatorname{Aff}_{\mathbb{Z}}[\boldsymbol{x}]^{2}$ the universality problem is undecidable. This contrasts the known results on the emptiness problem as we showed that the emptiness problem is undecidable for maps over $\operatorname{Aff}_{\mathbb{Z}}[\boldsymbol{x}]^{3}$ and NP-hard for maps over $\operatorname{Aff}_{\mathbb{Z}}[\boldsymbol{x}]^{2}$. The model is connected to blind counter automata that have been extensively studied in the past. 
In [2] the authors study the zeroness problem, which is essentially the universality problem we consider in this section. The main difference is that our model is nondeterministic, while in [2] the automaton is deterministic. The authors show that the zeroness problem is ACKERMANN-complete $\mathrm{e}^{5}$.

Let us define the language acceptors more precisely. Given $G \subseteq \Sigma \times F$, where $F \subseteq \mathbb{Z}[\boldsymbol{x}]^{n}$ is an $n$-dimensional polynomial map, $\boldsymbol{x}_{0}$ and $\boldsymbol{x}_{f}$ the initial and target vectors, respectively, and $\Sigma$ is an alphabet, a word $a_{i_{1}} a_{i_{2}} \cdots a_{i_{k}}$ is accepted by $G$, i.e., is in $L(G)$, if there exists $\pi=\left(\left(a_{i_{1}}, f_{i_{1}}\right),\left(a_{i_{2}}, f_{i_{2}}\right), \ldots,\left(a_{i_{k}}, f_{i_{k}}\right)\right)$ such that $f_{i_{k}}\left(\cdots f_{i_{2}}\left(f_{i_{1}}\left(\boldsymbol{x}_{0}\right)\right) \cdots\right)=\boldsymbol{x}_{f}$.

Theorem 13. The universality problem for maps over $A f_{\mathbb{Z}}[\boldsymbol{x}]^{2}$ is undecidable.

Proof. Let $\mathcal{A}^{\gamma}$ be an integer weighted automaton over alphabet $\Sigma$ for which the universality problem is undecidable [9]. Let $m$ be the number of states of $\mathcal{A}^{\gamma}$ and let us enumerate them as $q_{0}, \ldots, q_{m-1}$ such that $q_{0}$ is the initial state and $q_{m-1}$ is the final state ${ }^{6}$. The idea is to encode $\mathcal{A}^{\gamma}$ into maps in such way that the second dimension is used to simulate the state transitions of the automaton using Lemma 3. Let $\left(q_{i}, a, q_{j}, z\right)$ be a transition of $\mathcal{A}^{\gamma}$ with which the automaton reads letter $a \in \Sigma$ in state $q_{i}$, changes its state to $q_{j}$ and adds $z$ to the weight, i.e., applies function $f(x)=x+z$. The corresponding two-dimensional affine function is $\left(a,\left(x_{1}+z, m \cdot x_{2}+j-m \cdot i\right)\right)$. Now, a word $w \in \Sigma^{*}$ is accepted by the map if and only if the register values $(0, m-1)$ are reachable from $(0,0)$ while reading word $w$. It is clear that the map accepts $w$ if and only if the automaton accepts $w$. That is, it is undecidable whether the map accepts every finite word.

We further investigate the properties of the reachability sets of different maps. Let us first define a reachability set. Let $F \subseteq \mathbb{Z}[\boldsymbol{x}]^{n}$ be a map over $\mathbb{Z}[\boldsymbol{x}]^{n}$ and let $\boldsymbol{x}_{0} \in \mathbb{Z}^{n}$ be the initial value. The reachability set of $F$ is defined iteratively:

$$
\begin{aligned}
\operatorname{Reach}_{0}(F) & =\left\{\boldsymbol{x}_{0}\right\}, \\
\operatorname{Reach}_{i}(F) & =\left\{f(\boldsymbol{x}) \mid \boldsymbol{x} \in \operatorname{Reach}_{i-1}(F), f \in F\right\}, \\
\operatorname{Reach}(F) & =\bigcup_{i=0}^{\infty} \operatorname{Reach}_{i}(F) .
\end{aligned}
$$

Next we show that the intersection non-emptiness problem is undecidable for $\operatorname{Aff}_{\mathbb{Z}}[\boldsymbol{x}]^{2}$ using the PCP. That is, for $F, G \subseteq \operatorname{Aff}_{\mathbb{Z}}[\boldsymbol{x}]^{2}$ whether $\operatorname{Reach}(F) \cap$ $\operatorname{Reach}(G) \neq \emptyset$. The idea is for $F$ (resp. $G$ ) to construct tertiary representation of words $u_{i}$ (resp. $v_{i}$ ) in the first dimension and the corresponding indices in the second. Then the intersection is non-empty if and only if it possible to construct the same word, i.e., the PCP has a solution.

\footnotetext{
${ }^{5}$ Ackermann is a complexity class containing decision problems solvable in time bounded by Ackermann function, which is computable but not primitive-recursive.

${ }^{6}$ The automata of [9] were defined without final states but the weighted automaton with undecidable universality problem was constructed in such a way that a non-empty word can be accepted only in a single state.
} 
Lemma 14. Let $F$ and $G$ be two-dimensional affine maps. It is undecidable whether the intersection of the respective reachability sets is empty or not.

By modifying the proof idea of the previous lemma, we show that the language intersection problem is also undecidable. The idea is to construct two maps each accepting indices of one of the components of the PCP instance. Then the intersection of the languages is non-empty if and only if there exists a sequence of indices such that both maps store the same tertiary representations.

Theorem 15. Let $F, G \subseteq \Sigma \times A f f_{\mathbb{Z}}[\boldsymbol{x}]^{2}$ and $\boldsymbol{x}_{0_{F}}, \boldsymbol{x}_{0_{G}}$ and $\boldsymbol{x}_{f_{F}}, \boldsymbol{x}_{f_{G}}$ be the respective initial and target values. It is undecidable whether the intersection of the respective languages is empty, that is, whether $L(F) \cap L(G)=\emptyset$ holds or not.

\section{References}

1. P. Bell and I. Potapov. On undecidability bounds for matrix decision problems. Theor. Comput. Sci., 391(1-2):3-13, 2008.

2. M. Benedikt, T. Duff, A. Sharad, and J. Worrell. Polynomial automata: Zeroness and applications. In LICS 2017, pp. 1-12, 2017.

3. V. D. Blondel, O. Bournez, P. Koiran, C. H. Papadimitriou, and J. N. Tsitsiklis. Deciding stability and mortality of piecewise affine dynamical systems. Theor. Comput. Sci., 255(1-2):687-696, 2001.

4. V. D. Blondel and J. N. Tsitsiklis. A survey of computational complexity results in systems and control. Automatica, 36(9):1249-1274, 2000.

5. P. Collet and J.-P. Eckmann. Iterated Maps on the Interval as Dynamical Systems. Birkhuser Boston, 2009.

6. A. Finkel, S. Göller, and C. Haase. Reachability in register machines with polynomial updates. In MFCS 2013, vol. 8087 of LNCS, pp. 409-420, 2013.

7. J. Frøyland. Introduction to Chaos and Coherence. CRC Press, 1992.

8. C. Haase and S. Halfon. Integer vector addition systems with states. In RP 2014, vol. 8762 of $L N C S$, pp. 112-124, 2014.

9. V. Halava and T. Harju. Undecidability in integer weighted finite automata. Fundam. Inform., 38(1-2):189-200, 1999.

10. J. E. Hopcroft and J. D. Ullman. Introduction to Automata Theory, Languages and Computation. Addison-Wesley Publishing Company, 1979.

11. T. Neary. Undecidability in binary tag systems and the Post correspondence problem for five pairs of words. In STACS 2015, vol. 30 of LIPIcs, pp. 649-661, 2015.

12. R. Niskanen. Reachability problem for polynomial iteration is PSPACE-complete. In RP 2017, vol. 10506 of LNCS, pp. 132-143, 2017.

13. J. Reichert. Reachability Games with Counters: Decidability and Algorithms. Doctoral thesis, Laboratoire Spécification et Vérification, ENS Cachan, France, 2015.

14. T. Skolem. Ein Verfahren zur Behandlung gewisser exponentialer Gleichungen und diophantischer Gleichungen. 8. Skand. Mat. Kongr. Stockholm, pp. 163-188, 1934.

15. N. K. Vereshchagin. The problem of appearance of a zero in a linear recurrence sequence. Mat. Zametki, 38(2):609-615, 1985. 